\title{
COMPARISON OF 1998 AND 1999 LEONID LIGHT CURVE MORPHOLOGY AND METEOROID STRUCTURE
}

\author{
IAN S. MURRAY AND MARTIN BEECH \\ Department of Physics, University of Regina, Regina, Saskatchewan, \\ CANADA S4S OA2 \\ E-mail:murrayli@mail.uregina.ca
}

MICHAEL J. TAYLOR

Space Dynamics Laboratory, Utah State University, Logan, Utah 84322-4145, USA

PETER JENNISKENS

SETI Institute, NASA ARC, Mail Stop 239-4, Moffett Field, CA 94035, USA

and

ROBERT L. HAWKES

Mount Allison University, Physics Department, 67 York St., Sackville, New Brunswick, CANADA E4L IE6

(Received 27 June 2000; Accepted 14 July 2000)

\begin{abstract}
Photometric low-light level video observations of 1999 Leonid storm meteors have been obtained from two airborne platforms during the Leonid multiinstrument aircraft campaign (Leonid MAC). The 1999 Leonid light curves tend to be skewed towards the end point of the trajectory, while the 1998 Leonid light curves were not. The variation in the light curves from 1998 and 1999 can be explained as an overall reduction in the mass distribution index, $\alpha$ from $\sim 1.95$ in 1998 to -1.75 in 1999. We have interpreted this behaviour as being either indicative of a gradual loss of the "glue" that keeps the grains together, or the fact that the meteoroids sampled in 1998 had a different morphological structure to those sampled in 1999. The early fragmentation of a dustball meteoroid results in a light curve that peaks sooner than that predicted by classical single body ablation theory.
\end{abstract}

Keywords: Comets, dust, Leonids 1999, lightcurves, meteoroids, meteors 
Draconid, and $\alpha$-Capricornid meteor showers. Here the dustball model has been expanded to include a description of the mass distribution of grains in a meteoroid. A distribution in the masses of the constituent grains results in an overall light curve that is broader and is earlier skewed than that produced by a classical light curve (Campbell, 1999).

During the 1998 Leonid Multi-Instrument Aircraft Campaign, we measured light curves of a sample of Leonid meteors and described the asymmetry of the light curves, many of which were skewed towards the early part of the trajectory (Murray et al., 1999). We have now measured a sample of light curves from the 1999 Leonid storm under similar conditions and find a quite different behaviour.

\section{Experimental Observations}

The experimental conditions in this study were similar to those during the 1998 Leonid MAC (Murray et al., 1999). Two co-aligned intensified cameras were pointed at a constant elevation angle of $75^{\circ}$ out of a high window port on the FISTA (Flying Infrared Signature Technologies Aircraft). This time, however, both cameras were synchronised using an $\mathrm{AC}$ coupling feature of the individual camera systems; this allowed for accurate synchronous frame information to be obtained. Also, one of the two cameras, designated $\mathrm{N}$, was equipped with a narrow band (bandwidth: $50 \%$ transmission at $8.96 \mathrm{~nm}$ and $10 \%$ at $13.03 \mathrm{~nm}$ ) sodium filter centred at $589.50 \mathrm{~nm}$. With peak transmittance of $64 \%$, the filtered camera reached a limiting apparent stellar magnitude of approximately +4.0 .

In parallel with these measurements, narrow field observations were made from the ARIA (Advanced Ranging Instrumentation Aircraft) using two co-aligned Xybion intensified video cameras mounted at an elevation of $\sim 30^{\circ}$ on the starboard side of the aircraft. One camera, type RG-350 fitted with a GEN III image intensifier, had a spectral range of $\sim 350-900 \mathrm{~nm}$ and was fitted with a variety of filters during the night of the storm including two narrow band interference filters; one centered on the magnesium emission at $\sim 520 \mathrm{~nm}$ and the other on the sodium emission at $589 \mathrm{~nm}$. Both filters had a bandwidth of $10 \mathrm{~nm}$ (full-width at half maximum) and a peak transmission of $\sim 50 \%$. This imager was fitted with a $75 \mathrm{~mm}, \mathrm{f} / 1.4$ lens (field of view $8^{\circ} \times 7^{\circ}$ ) and well over 200 meteors were recorded at various emission wavelengths of which 43 


\section{TABLE I}

\begin{tabular}{|c|c|c|c|c|c|c|}
\hline $\begin{array}{l}\text { Time } \\
\text { (UT) }\end{array}$ & $\begin{array}{l}\text { Maximum } \\
\text { Lumin. } \\
\left(0^{M}\right)\end{array}$ & $\begin{array}{l}\text { Photometric } \\
\text { Mass (kg) }\end{array}$ & $F_{\text {averes }}$ & $\mathrm{B}$ & $\mathbf{M}$ & $E$ \\
\hline $00: 04: 07$ & 3.2 & $1.1 \times 10^{-6}$ & 0.76 & 0 & 1 & 1 \\
\hline $00: 21: 21$ & 4.5 & $1.6 \times 10^{-7}$ & 0.51 & 1 & 1 & 1 \\
\hline $00: 47: 39$ & 4.4 & $2.9 \times 10^{-9}$ & 0.74 & 1 & 1 & 1 \\
\hline $00: 47: 47$ & 5.7 & $3.3 \times 10^{-2}$ & 0.74 & 0 & 1 & 1 \\
\hline $00: 52: 16$ & 3.5 & $1.1 \times 10^{-6}$ & 0.79 & 1 & 1 & 1 \\
\hline $00: 56: 21$ & 3.6 & $6.5 \times 10^{7}$ & 0.37 & 1 & 1 & 1 \\
\hline $01: 07: 36$ & 3.9 & $3.8 \times 10^{-7}$ & 0.65 & 1 & 1 & 1 \\
\hline $01: 10: 57$ & 4.4 & $1.9 \times 10^{7}$ & 0.57 & 1 & 1 & 1 \\
\hline $01: 17: 28$ & 5.8 & $2.9 \times 10^{-3}$ & 0.79 & $i$ & 1 & 1 \\
\hline $01: 22: 00$ & 3.5 & $4.3 \times 10^{7}$ & 0.58 & 1 & 1 & 0 \\
\hline $01: 29: 00$ & 3.6 & $4.8 \times 10^{7}$ & 0.56 & $i$ & $i$ & 1 \\
\hline $01: 32: 13$ & 4.2 & $2.6 \times 10^{-7}$ & 0.57 & 1 & 1 & I \\
\hline $01: 37: 01$ & 4.4 & $1.5 \times 10^{-7}$ & 0.49 & 1 & 1 & 1 \\
\hline $01: 37: 33$ & 3.8 & $3.2 \times 10^{-7}$ & 0.52 & 0 & 1 & 1 \\
\hline $01: 38: 40$ & 5.7 & $6.1 \times 10^{2}$ & 0.54 & 1 & 1 & 1 \\
\hline $01: 38: 48$ & 3.8 & $3.8 \times 10^{-7}$ & 0.63 & 0 & 1 & 1 \\
\hline $01: 41: 38$ & 3.8 & $4.0 \times 10^{-2}$ & 0.81 & 1 & 1 & 0 \\
\hline $01: 43: 14$ & 5.0 & $1.0 \times 10^{-7}$ & 0.68 & 1 & 1 & 1 \\
\hline $01: 45: 57$ & 3.9 & $2.8 \times 10^{-7}$ & 0.63 & 0 & i & i \\
\hline $01: 49: 48$ & 6.0 & $2.6 \times 10^{-2}$ & 0.76 & 0 & 1 & $i$ \\
\hline $01: 51: 03$ & 4.2 & $1.5 \times 10^{2}$ & 0.65 & 1 & I & 1 \\
\hline $01: 51: 05$ & 4.5 & $1.9 \times 10^{-7}$ & 0.84 & 1 & 1 & 1 \\
\hline $01: 53: 47$ & 4.1 & $5.2 \times 10^{7}$ & N.V. & 1 & 1 & 0 \\
\hline $01: 54: 36$ & 2.7 & $1.4 \times 10^{-4}$ & 0.56 & 1 & 1 & 1 \\
\hline $01: 57: 57$ & 4.0 & $3.1 \times 10^{\circ}$ & 0.71 & $i$ & $i$ & 0 \\
\hline $01: 58: 59$ & 4.0 & $2.8 \times 10^{-7}$ & 0.46 & 1 & 1 & I \\
\hline $02: 01: 45$ & 3.6 & $6.7 \times 10^{-7}$ & 0.72 & 1 & $i$ & $i$ \\
\hline $02: 10: 38$ & 2.9 & $1.1 \times 10^{-1}$ & 0.54 & 1 & 1 & $i$ \\
\hline $02: 11: 52$ & 2.8 & $1.3 \times 10^{-6}$ & 0.69 & 1 & 1 & 1 \\
\hline $02: 12: 49$ & 3.0 & $9.5 \times 10^{-7}$ & 0.69 & 0 & 1 & 1 \\
\hline $02: 13: 41$ & 3.2 & $1.0 \times 10^{-6}$ & 0.59 & 1 & 1 & 1 \\
\hline $02: 15: 46$ & 4.2 & $1.5 \times 10^{-7}$ & 0.56 & 1 & 1 & 1 \\
\hline $02: 16: 22$ & 3.8 & $3.9 \times 10^{7}$ & 0.58 & 1 & 1 & 1 \\
\hline $02: 18: 20$ & 6.1 & $2.3 \times 10^{-n}$ & 0.14 & 1 & 1 & 1 \\
\hline $02: 19: 27$ & 5.0 & $9.2 \times 10^{8}$ & 0.51 & $i$ & $i$ & $i$ \\
\hline $02: 21: 29$ & 4.8 & $7.9 \times 10^{-8}$ & 0.30 & 0 & 1 & I \\
\hline $02: 22: 42$ & 3.5 & $5.4 \times 10^{-7}$ & 0.57 & 1 & 1 & 0 \\
\hline $02: 25: 50$ & 3.7 & $4.9 \times 10^{-1}$ & N.V. & 1 & 1 & 1 \\
\hline $02: 32: 22$ & 3.6 & $4.1 \times 10^{-7}$ & 0.40 & 1 & 1 & I \\
\hline $02: 56: 12$ & 2.6 & $1.5 \times 10^{-4}$ & 0.67 & 1 & 1 & 1 \\
\hline $02: 56: 27$ & 3.5 & $6.5 \times 10^{-7}$ & 0.82 & 1 & I & 1 \\
\hline 03:01:42 & 3.8 & $3.0 \times 10^{-7}$ & 0.73 & 1 & 1 & 1 \\
\hline 03:09:59 & 4.3 & $1.8 \times 10^{-7}$ & 0.63 & 1 & 1 & 1 \\
\hline $03: 31: 26$ & 3.6 & $4.8 \times 10^{7}$ & 0.42 & 0 & 1 & 1 \\
\hline $04: 07: 10$ & 5.5 & $4.8 \times 10^{-1}$ & 0.54 & 1 & 1 & I \\
\hline $04: 12: 45$ & 3.7 & $3.5 \times 10^{-7}$ & 0.71 & 1 & 1 & 1 \\
\hline $04: 21: 59$ & 3.6 & $5.4 \times 10^{7}$ & 0.72 & $i$ & $i$ & 1 \\
\hline $04: 52: 00$ & 3.7 & $3.6 \times 10^{\prime}$ & 0.55 & 1 & 1 & 1 \\
\hline
\end{tabular}


where $t_{\max }$ is the time of light curve maximum and $t_{B \Delta m}$ and $t_{E_{\Delta M}}$ are the beginning and end times at which the brightness is $\Delta \mathrm{m}$ magnitudes fainter than the maximum. The F-values were calculated at magnitude intervals of $\Delta \mathrm{m}$ equal to $0.25,0.50,0.75,1.0$ and 1.25 fainter than maximum brightness. The F-yalues where then averaged to give the values listed in Table $\mathrm{I}$.

\section{TABLE II}

\begin{tabular}{lllllll} 
& Camera System & $F_{0.25}^{*}$ & $F_{0.50}$ & $F_{0.75}$ & $F_{1.00}$ & $F_{1.25}$ \\
\hline Mean $50 \mathrm{~mm} \mathrm{1999}$ & 0.58 & 0.61 & 0.62 & 0.63 & 0.61 \\
Std. Dev. & 0.17 & 0.15 & 0.15 & 0.14 & 0.15 \\
Mean $50 \mathrm{~mm} \mathrm{1998}$ & 0.46 & 0.45 & 0.48 & 0.47 & 0.49 \\
Std. Dev. & 0.20 & 0.18 & 0.14 & 0.15 & 0.14 \\
\hline
\end{tabular}

*) Mean F-values for the Leonid light curves sampled in 1998 (penultimate row) and 1999 (first row).

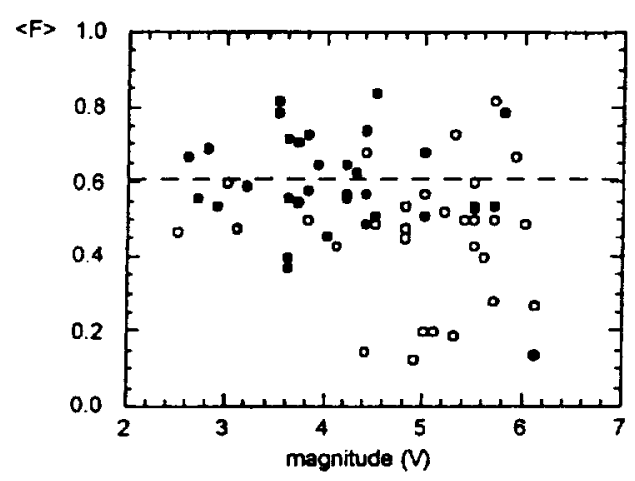

Figure 2. The F-parameter as a function of peak brightness of the meteors. Dashed line shows the mean $F$ value for a classical light curve while $(0)$ and $(\bullet)$ designate 1998 and 1999 data points.

The result of averaging the F-values for all of the sampled light curves (Table II) is a mean of 0.61 , essentially the value expected for a classical light curve with a peak occurring towards the end of the trajectory. A perfectly symmetric light curve will have an F-value of 0.5 for all $\Delta \mathrm{m}$; a light curve with an early maximum will have $F<0.5$; a late maximum will have $\mathrm{F}>0.5$. However, this result is somewhat misleading since the 


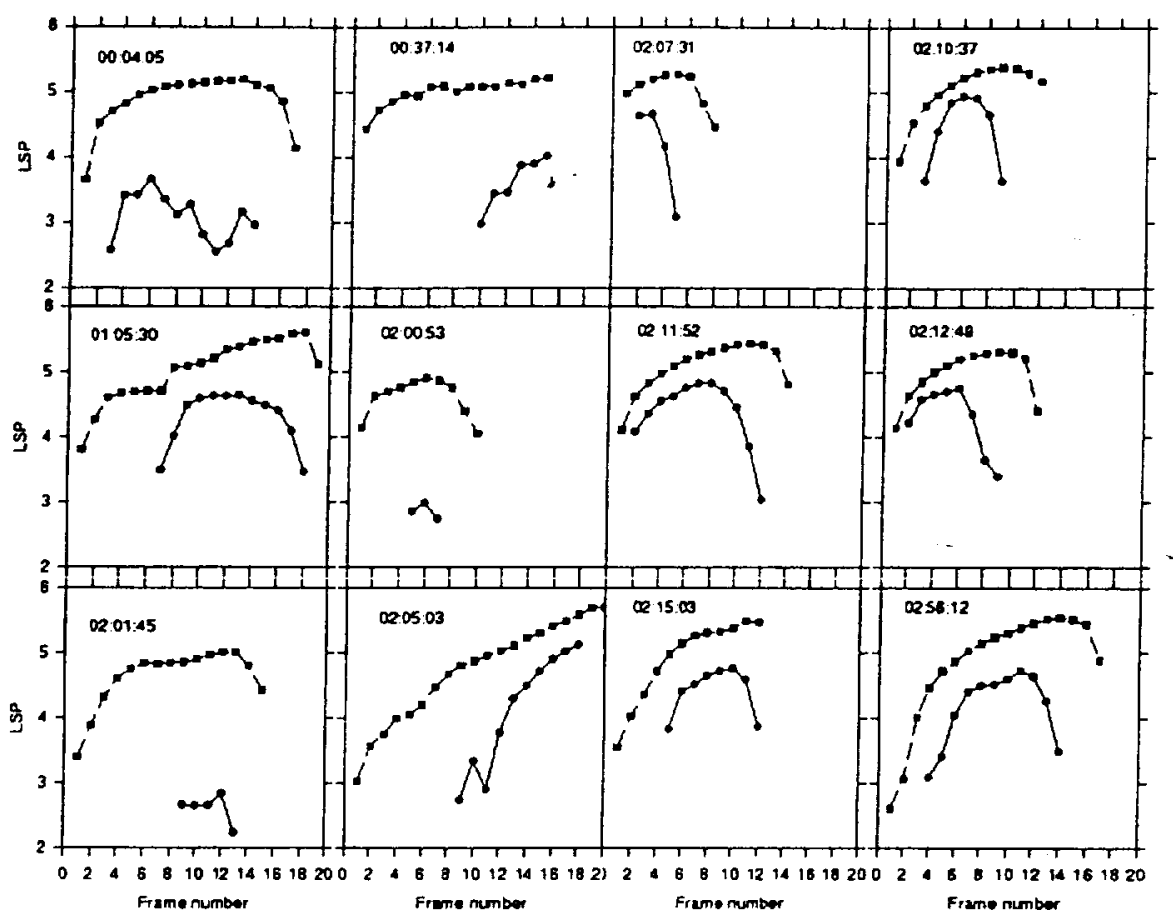

Figure 5. Comparison of the sodium filtered and unfiltered light curves for 12 Leonid meteors. In each diagram the $\mathrm{Na}$ light curve is always the lower of the two displayed. In this diagram the vertical axis corresponds to the log sum pixel (LSP) count and the horizontal axis is a relative time scale showing individual sequential video frames. Each frame is separated by a time interval of $1 / 30^{\text {th }}$ of a second. The filled circles and squares are individual data points.

Figure 2 shows the skew (averaged F-values) of both 1998 and 1999 light curves as a function of the peak brightness. A trend towards lower $F$ values for the 1998 light curves is apparent in Figure 2. Seven light curves from 1998 have $F<0.4$, while only 2 from 1999 are smaller than 0.4 . Four light curves from 1998 have $F>0.6$ while 14 of the 1999 light curves have averaged F-values larger than 0.6 . The difference in the light curves also shows up in the calculated photometric masses. For a given peak brightness, the 1998 meteors have a smaller integrated intensity (photometric mass) as shown in Figure 3. The dashed line in Figure 3 shows the expected relationship if all the meteor light curves are identical and a constant fraction of the kinetic energy is transformed into light (this assumption implies $\log M \sim 0.4 m_{v}$ ). When plotting the F- 
their overall morphology. As first indicated by Borovicka et al. (1999) one might expect to find systematic differences between the light curves observed through sodium and magnesium filters. This situation can arise because sodium and magnesium reside in different host phases in IDPs (Rietmeijer, 1998; 1999; 2000) and meteorites (Papike, 1998). To date 14 complete ( 5 magnesium filtered and 9 sodium filtered) Leonid light curves have been examined (see the scaled sequence of light curves in Figure 6).
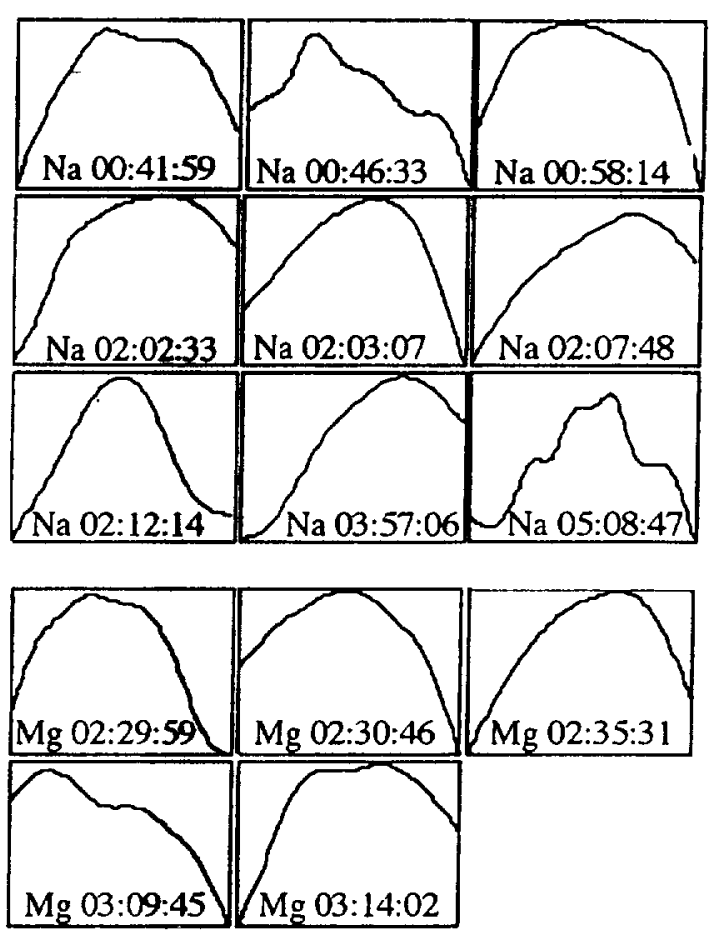

Figure 6. Nine calibrated filtered $\mathrm{Na}$ (top) and five $\mathrm{Mg}$ (bottom) light curves from the ARIA observations. As with the earlier presented light curves, they are plotted with relative scales to show overall morphologies.

We find that, in general, the morphology of the filtered light curves is similar to those shown by the non-filtered observations (see Figures 5). Some sodium light curves show significant intensity variations along the meteor trajectory. Although low number statistics prevail, F-values were computed for the ARIA filtered light curves at log sum pixel intervals 
mass distribution index $(\alpha)$. It can be seen that 'flat topped' light curves require a mass distribution index $\alpha \sim 1.8$.

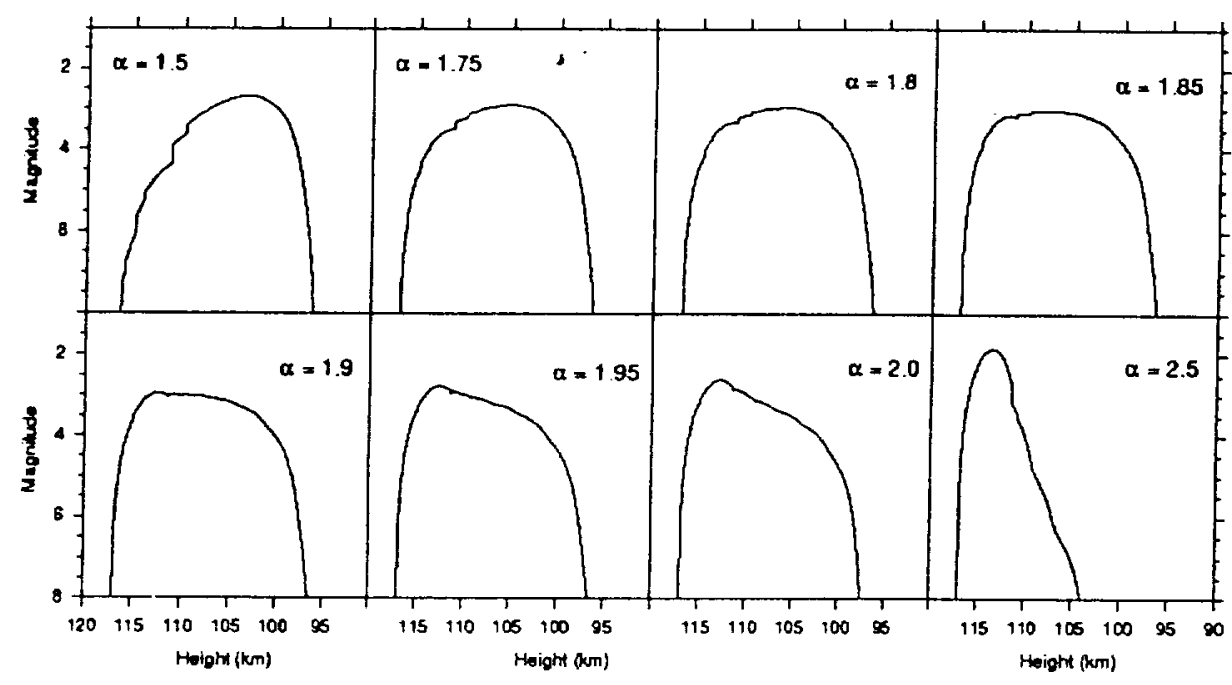

Figure 7: Synthesised light curves for a $10^{-6} \mathrm{~kg}$ Leonid meteoroids for a range of grain size distributions.

For small values of $\alpha<1.5$ the synthesised light curve approaches the classical light curve of the largest mass grain. Likewise the synthesised light curves constructed with $\alpha>2.5$ would approach that of the classical light curve of the lowest mass grain. For $1.5<\alpha<2.5$, a range of variable light curve morphologies are realised. We see, for example, that when $\alpha \sim 1.5$ the light curve has a near linear increase to its maximum and is late skewed. For $\alpha \sim 1.85$ the light curve is very nearly 'flat topped' and symmetrical about the maximum. For $\alpha>1.9$ the light curve has an early peak and displays a near linear decrease of magnitude with time after the maximum. We see also from Figure 7 that there is a systematic shift in the height of the light curve maximum as $\alpha$ increases from 1.5 to 2.5 . For $\alpha=1.5$ the maximum is at $103.4 \mathrm{~km}$ altitude, at $\alpha=$ 2.5 the maximum is at $113.4 \mathrm{~km}$ altitude (assuming a zenith angle of 45 degrees). The change in height of the light curve maximum, as a function of the mass distribution index, is at its most dramatic for $1.8<\alpha<1.9$. In this range the height of maximum increases by some 7 kilometres. In 


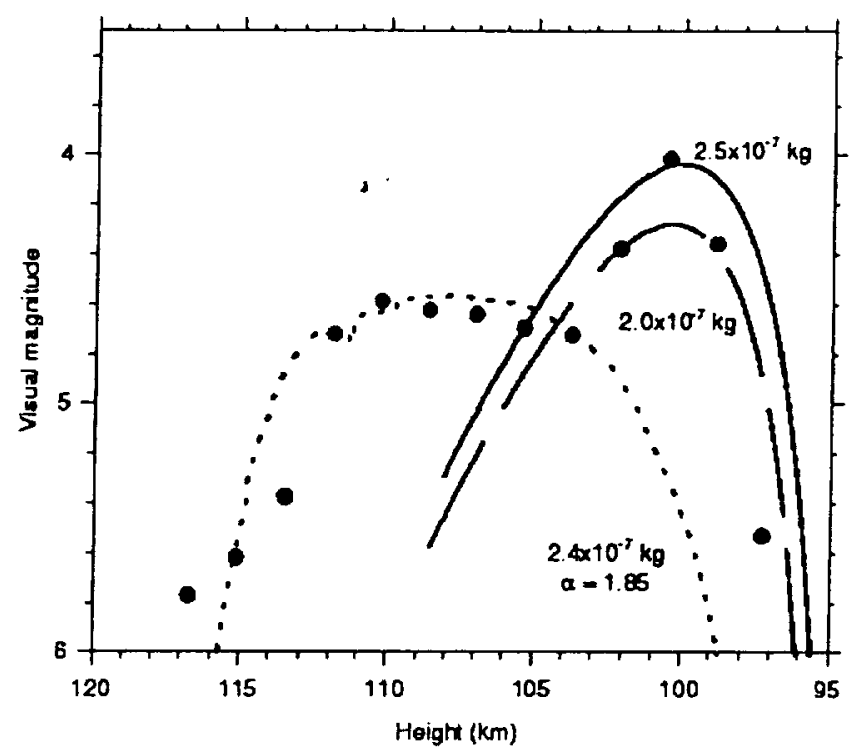

Figure 8. Composite light curve model for the 'humped' Leonid meteor observed at 01:57:54 UT, 1999 November 17. The dots correspond to the derived magnitudes. The dashed line shows the synthesised light curve for a 2.4 $\times 10^{-7} \mathrm{~kg}$ meteoroid with $\alpha=1.85$. The solid and broken-solid lines correspond to the classical light curves for 2.0 and $2.5 \times 10^{-7} \mathrm{~kg}$ meteoroids.

\section{Discussion}

The apparently higher mass distribution index $\alpha$ for the constituent grains of the 1998 Leonid meteoroids is highly interesting. One reason for this apparent enhancement could be that the 1998 meteoroids have a significantly different ejection age than the meteoroids sampled in other years. While the 1999 Leonid shower was predominantly composed of debris ejected in 1899 (McNaught and Asher, 1999), the material sampled in 1998 was ejected from comet 55P/Tempel-Tuttle several perihelion passages before 1899 (Asher et al., 1999; Jenniskens and Betlem, 2000). If older grains dominate the 1998 Leonid sample set it would imply that meteoroids become progressively more fragmented over time during their exposure to the interplanetary environment. This effect may be mitigated through repeated heating and cooling episodes associated with returns to perihelion, and possibly the "glue" that holds the grains together may be gradually lost or made less sticky by UV 
TABLE I

\begin{tabular}{|c|c|c|c|c|c|c|}
\hline Time (UT) & $\begin{array}{l}\text { Maximum } \\
\text { Luminosity } \\
\left(0^{M}\right)\end{array}$ & $\begin{array}{l}\text { Photometric } \\
\text { Mass (kg) }\end{array}$ & $\overline{F_{\text {monsers }}}$ & B & $\mathbf{M}$ & $\bar{E}$ \\
\hline 00:04:07 & 3.2 & $11: 1 \times 10^{-6}$ & 0.76 & 0 & 1 & 1 \\
\hline $00: 21: 21$ & 4.5 & $1.6 \times 10^{-1}$ & 0.51 & 1 & 1 & 1 \\
\hline $00: 47: 39$ & 4.4 & $2.9 \times 10^{-7}$ & 0.74 & 1 & 1 & 1 \\
\hline $00: 47: 47$ & 5.7 & $3.3 \times 10^{-3}$ & 0.74 & 0 & 1 & 1 \\
\hline $00: 52: 16$ & 3.5 & $1.1 \times 10^{-6}$ & 0.79 & 1 & 1 & 1 \\
\hline 00:56:21 & 3.6 & $6.5 \times 10^{-7}$ & 0.37 & 1 & 1 & 1 \\
\hline $01: 07: 36$ & 3.9 & $3.8 \times 10^{-7}$ & 0.65 & 1 & 1 & 1 \\
\hline 01:10:57 & 4.4 & $1.9 \times 10^{7}$ & 0.57 & 1 & 1 & 1 \\
\hline 01:17:28 & 5.8 & $2.9 \times 10^{-8}$ & 0.79 & 1 & 1 & 1 \\
\hline $01: 22: 00$ & 3.5 & $4.3 \times 10^{7}$ & 0.58 & 1 & 1 & 0 \\
\hline $01: 29: 00$ & 3.6 & $4.8 \times 10^{-7}$ & 0.56 & 1 & 1 & 1 \\
\hline 01:32:13 & 4.2 & $2.6 \times 10^{-7}$ & 0.57 & 1 & 1 & 1 \\
\hline $01: 37: 01$ & 4.4 & $1.5 \times 10^{-7}$ & 0.49 & 1 & 1 & 1 \\
\hline 01:37:33 & 3.8 & $3.2 \times 10^{-1}$ & 0.52 & 0 & 1 & 1 \\
\hline 01:38:40 & 5.7 & $6.1 \times 10^{-3}$ & 0.54 & 1 & 1 & 1 \\
\hline 01:38:48 & 3.8 & $3.8 \times 10^{7}$ & 0.63 & 0 & 1 & 1 \\
\hline 01:41:38 & 3.8 & $4.0 \times 10^{-7}$ & 0.81 & 1 & 1 & 0 \\
\hline 01:43:14 & 5.0 & $1.0 \times 10^{-7}$ & 0.68 & 1 & $i$ & 1 \\
\hline $01: 45: 57$ & 3.9 & $2.8 \times 10^{-1}$ & 0.63 & 0 & 1 & 1 \\
\hline $01: 49: 48$ & 6.0 & $2.6 \times 10^{-3}$ & 0.76 & 0 & $i$ & 1 \\
\hline 01:51:03 & 4.2 & $1.5 \times 10^{7}$ & 0.65 & 1 & 1 & i \\
\hline 01:51:05 & 4.5 & $1.9 \times 10^{7}$ & 0.84 & i & $i$ & i \\
\hline 01:53:47 & 4.1 & $5.2 \times 10^{-7}$ & N.V. & 1 & 1 & 0 \\
\hline $01: 54: 36$ & 2.7 & $1.4 \times 10^{-6}$ & 0.56 & 1 & 1 & i \\
\hline $01: 57: 57$ & 4.0 & $3.1 \times 10^{-7}$ & 0.71 & 1 & 1 & 0 \\
\hline 01:58:59 & 4.0 & $2.8 \times 10^{7}$ & 0.46 & 1 & 1 & 1 \\
\hline 02:01:45 & 3.6 & $6.7 \times 10^{-7}$ & 0.72 & 1 & 1 & 1 \\
\hline $02: 10: 38$ & 2.9 & $1.1 \times 10^{-6}$ & 0.54 & 1 & 1 & 1 \\
\hline 02:11:52 & 2.8 & $1.3 \times 10^{-6}$ & 0.69 & 1 & 1 & 1 \\
\hline $02: 12: 49$ & 3.0 & $9.5 \times 10^{-7}$ & 0.69 & 0 & $i$ & 1 \\
\hline 02:13:41 & 3.2 & $1.0 \times 10^{-6}$ & 0.59 & 1 & $i$ & $i$ \\
\hline $02: 15: 46$ & 4.2 & $1.5 \times 10^{7}$ & 0.56 & 1 & 1 & 1 \\
\hline $02: 16: 22$ & 3.8 & $3.9 \times 10^{-7}$ & 0.58 & 1 & 1 & I \\
\hline $02: 18: 20$ & 6.1 & $2.3 \times 10^{-8}$ & 0.14 & 1 & 1 & 1 \\
\hline $02: 19: 27$ & 5.0 & $9.2 \times 10^{-8}$ & 0.51 & 1 & 1 & 1 \\
\hline $02: 21: 29$ & 4.8 & $7.9 \times 10^{-1}$ & 0.30 & 0 & 1 & 1 \\
\hline $02: 22: 42$ & 3.5 & $5.4 \times 10^{7}$ & 0.57 & 1 & 1 & 0 \\
\hline $02: 25: 50$ & 3.7 & $4.9 \times 10^{-7}$ & N.V. & 1 & 1 & 1 \\
\hline $02: 32: 22$ & 3.6 & $4.1 \times 10^{-7}$ & 0.40 & $i$ & $i$ & i \\
\hline 02:56:12 & 2.6 & $1.5 \times 10^{-6}$ & 0.67 & 1 & 1 & 1 \\
\hline 02:56:27 & 3.5 & $6.5 \times 10^{7}$ & 0.82 & 1 & 1 & 1 \\
\hline 03:01:42 & 3.8 & $3.0 \times 10^{-7}$ & 0.73 & $i$ & 1 & $i$ \\
\hline 03:09:59 & 4.3 & $1.8 \times 10^{-7}$ & 0.63 & $i$ & $i$ & $i$ \\
\hline $03: 31: 26$ & 3.6 & $4.8 \times 10^{-7}$ & 0.42 & 0 & $i$ & $i$ \\
\hline 04:07:10 & 5.5 & $4.8 \times 10^{-4}$ & 0.54 & 1 & 1 & 1 \\
\hline $04: 12: 45$ & 3.7 & $3.5 \times 10^{-7}$ & 0.71 & i & 1 & 1 \\
\hline 04:21:59 & 3.6 & $5.4 \times 10^{7}$ & 0.72 & 1 & 1 & 1 \\
\hline 04:52:00 & 3.7 & $3.6 \times 10^{7}$ & 0.55 & 1 & 1 & 1 \\
\hline
\end{tabular}

